\title{
Counting Coronavirus Disease-2019 (COVID-19) Cases: Case Definitions, Screened Populations and Testing Techniques Matter
}

David Koh, ${ }^{1,2}{ }^{2}$ D , FFOM, FRCPI, Anne Catherine Cunningham, ${ }_{B S C,}{ }^{P h D}$

\begin{abstract}
While counting cases of disease appears straightforward, there are issues to consider when enumerating disease counts during an epidemic. For example, for Coronavirus Disease-2019 (COVID-19), how is a case defined? Hubei province in China changed its case definition twice in a fortnight-from laboratory-confirmed cases to clinicallyconfirmed cases without laboratory tests, and back to laboratory-confirmed cases. This caused confusion in the reported number of cases. If a confirmed case requires laboratory testing, what is the population who are laboratory-tested? Due to limited laboratory testing capacity in the early phase of an emerging epidemic, only "suspected cases" are laboratory-tested in most countries. This will result in underdiagnosis of confirmed cases and also raises the question: how is a "suspect case" defined? With the passage of time and increased capability to perform laboratory tests, more people can be screened and the number of confirmed cases will increase. What are the technical considerations of laboratory testing? This includes specimen collection (variable collection methods), samples collected (upper or lower respiratory tract biospecimens), time of collection in relation to course of disease, different laboratory test methods and kits (not all of which may be standardised or approved by authorities such as the Food and Drug Administration). Are approved laboratory facilities and trained manpower available, and how are test results interpreted and false-negatives excluded? These issues will affect the accuracy of disease counts, which in turn will have implications on how we mount an appropriate response to the outbreak.
\end{abstract}

Ann Acad Med Singapore 2020;49:161-5

Key words: Diagnosis, Laboratory testing, Outbreak, Pandemic, Screening

\section{Introduction}

Several factors determine the number counts of Coronavirus Disease-2019 (COVID-19) - one of which is the case definition. It was reported on 13 February 2020 that there was an overnight steep increase in COVID-19 in China-15,152 cases and 254 deaths. This was caused by the broadening of the case definition (only in Hubei province and not the rest of China or elsewhere in the world) to include not only the 1820 cases confirmed by laboratory testing on that day, but also 13,332 clinically-confirmed cases (on the basis of chest imaging, without the need for laboratory tests), which had accumulated in the weeks since the start of the outbreak. ${ }^{1}$

The reason given for recognising clinically-confirmed cases was to allow clinicians to report cases more quickly without waiting for laboratory confirmation, for which there was a backlog. This would allow for prompt clinical care and public health responses such as contact tracing and quarantine. Thus, the spike of 14,000 cases in a single day had been caused by a change in case definition. The case definition in Hubei has since been changed again on 20 February 2020 to consider only laboratory-confirmed cases. ${ }^{2}$

However, even the use of a case definition (which requires laboratory confirmation of the disease) has limitations as it may lead to underdiagnosis of cases due to various reasons.

\section{Who Are Tested in the Population?}

During the early stages of an outbreak of a novel disease, there are limited test kits and facilities, so not every person can be tested. Testing has to be prioritised for suspected

${ }^{1}$ PAPRSB Institute of Health Sciences, Universiti Brunei Darussalam, Brunei Darussalam

${ }^{2}$ Saw Swee Hock School of Public Health, National University of Singapore, Singapore

Address for Correspondence: Prof David Koh, PAPRSB Institute of Health Sciences, Universiti Brunei Darussalam, Jalan Tungku Link, Bandar Seri Begawan BE1410, Brunei Darussalam.

Email: david.koh@ubd.edu.bn; david_koh@nuhs.edu.sg 
cases. Infected persons who seek medical attention, but who do not fall under the category of "suspected cases" would not be tested. Those who do not come forward to seek medical treatment will also not be tested.

The term "suspected cases" has to be clearly defined. This is usually based on a history of relevant travel or contact with infected persons and specific symptoms such as pneumonia-like symptoms. The definition of a "suspected case" would vary from country to country and during different time periods of the outbreak. For example, in the initial phase of the outbreak in China, "suspected cases" were likely to have moderate or severe illness, with criteria such as atypical pneumonia and/or acute respiratory distress used to define "suspected cases".

Internationally, countries primarily concerned about imported cases may have broader criteria for defining a suspect case. This might consist of clinical symptoms such as cough and fever, and recent travel history to an affected region; contact with a confirmed or probable case; or working in a healthcare facility that treats probable/confirmed cases. Such surveillance would be able to identify clinically milder cases. On the other hand, restricting testing only to persons with relevant travel, contact or work history may result in missing symptomatic cases that may have occurred through local transmission. ${ }^{3}$

\section{Relevant Travel History}

The definition of "relevant travel history" in a suspect case has evolved over time, due to the spread of COVID-19 to different countries, and the changing risk of infection for travel in newly affected countries. This is illustrated in the case of Singapore, where the case definition of a "suspect case" was repeatedly updated as the outbreak unfolded. The definition of a "suspect case" first appeared on 2 January 2020 in Singapore. It had 2 criteria: 1) a person with clinical signs and symptoms suggestive of pneumonia or severe respiratory infection with breathlessness and travel to or residence in Wuhan city within the last 14 days, or 2) a person with an acute respiratory illness of any degree of severity who, within 14 days before onset of illness, had close contact ${ }^{1}$ with a pneumonia case of unknown cause linked to the Wuhan cluster.

In view of the spread of COVID-19 to all parts of China, criterion number 1 was expanded to cover travel to any part of mainland China on 21 January 2020. This criterion was further extended to include travel to Daegu city or Cheongdo county, South Korea on 23 February 2020 because of the steep increase of cases in these locations. The list of countries was further enlarged on 3 March 2020 to include Iran, northern Italy, Japan and the Republic of Korea (and on 10 March, to any country outside of Singapore).

Criterion number 2 was expanded on 4 February 2020 to include "a person with an acute respiratory illness of any degree of severity who, within 14 days before onset of illness had" done any one of the following: 1) been to Hubei province (including Wuhan) or Zhejiang province (including Hangzhou), China; 2) been to a hospital in mainland China (this was further amended on 3 March to "a hospital in affected areas"); 3) had close contact with a case of COVID-19 infection; or 4) had frequent or close contact during work with recent travellers from mainland China (travel history in the last 14 days).

An expanded case definition for a "suspect case" will result in greater sensitivity for case detection and more number of cases being confirmed (while lowering the specificity). Having greater sensitivity will be important if cases have to be detected in order to institute early measures to contain the illness. Conversely, restricting the case definition may result in increased specificity, but will reduce the sensitivity of case detection and number of cases detected.

\section{Symptoms of COVID-19}

The symptoms that characterise COVID-19 can vary and some patients can even be asymptomatic. For example, gastrointestinal symptoms initially occur in about $10 \%$ of cases, and this is often not listed as a symptom that would define a suspect case. It was reported that a patient who initially presented with gastrointestinal symptoms was not suspected to have the illness and initially admitted to a surgical ward. This patient infected over 10 healthcare workers. ${ }^{4}$

A large Chinese case series $(n=72,314)$ has shown that $1.2 \%(n=889)$ of cases are asymptomatic. ${ }^{5}$ Another case report ${ }^{6}$ has demonstrated an asymptomatic carrier transmission of COVID-19. However, much is still unknown about the asymptomatic transmission of the disease and as such, many countries are currently only testing patients who are symptomatic.

\section{Should "Confirmed Cases" or "Probable Cases" Be Counted?}

An internationally standard and rigorous case definition of a "confirmed case" will allow for comparison of case numbers in different parts of the world, or within a country during the course of an epidemic. This will assist in the calibration of an appropriate public health response at different stages of a disease outbreak.

On the other hand, a "probable case" is usually less rigorously defined, and the definition may vary from country to country. While this makes meaningful comparison of case numbers from various locations less feasible, it has its uses. For example, the introduction of a clinical case definition in Hubei province on 13 February 2020 resulted in a spike of cases in 1 day, and rendered comparisons with other Chinese provinces and the rest of the world invalid. However, the change in case definition in Hubei 
was in response to delays in confirming the diagnosis with laboratory testing, due to inability of laboratory services to cope with the surge in demand for testing. Early confirmation of cases was needed, so that preventive control measures such as isolation, contact tracing, risk communication and quarantine could be instituted immediately since delays in these actions could prove detrimental. This is an important consideration in situations when there are few diagnostic kits available or in low- or middle-income countries where testing facilities can be limited.

\section{What Biological Sample is Collected?}

Samples required for initial diagnostic testing include both upper and lower respiratory tract samples ${ }^{7}$ such as single or combined nose/throat swab, nasopharyngeal aspirate or sputum. A serum sample may also be useful for acute serological testing to rule out other causes of viral pneumonia (e.g. influenza, H1N1, H5N1, H7N9). Commercially produced serological tests for COVID-19 are beginning to be available. However, it would be useful to determine the levels of specific immunoglobulin M (IgM) and immunoglobulin $\mathrm{G}(\mathrm{IgG})$ antibodies (e.g. via enzymelinked immunosorbent assay [ELISA]) and their ability to neutralise the virus (e.g. via microneutralisation assays).

The sample collection technique - nasal or throat swab, wash or aspirate - can vary and affect the amount of virus collected. The type of biological samples taken for testing also have different diagnostic yields. For example, lower respiratory specimens obtained from sputum, lower respiratory tract aspirate or bronchioalveolar lavage have higher diagnostic value than upper respiratory specimens. The World Health Organization recommends that if initial testing is negative in a patient who is strongly suspected to have COVID-19, the patient should be resampled and specimens collected from multiple respiratory tract sites. Additional specimens such as blood, urine and stool might also be collected to assess virus presence or shedding. ${ }^{8}$

\section{When Are Biospecimens Collected?}

The timing of the test is important. In the early stages of the disease, the viral load may be lower and thus might not be detectable. According to the Centers for Disease Control and Prevention (CDC), "In the early stages of infection, it is possible the virus will not be detected". It adds that "a negative test for a sample collected while a person has symptoms likely means [COVID-19] is not causing their illness" (which describes a true negative result in a symptomatic person).

A woman travelled from Wuhan to Anyang on 10 January 2020 and visited several relatives. When 5 of her relatives developed COVID-19, she was isolated and tested for coronavirus. The woman tested negative on 26 January 2020 , but a follow-up test on 28 January 2020 was positive.
Computed tomography scans of the chest on 27 and 31 January 2020 showed no significant abnormalities and as of 11 February 2020, she had no elevated temperature or self-reported fever and no gastrointestinal or respiratory symptoms, including cough and sore throat. ${ }^{6}$ This woman represents either an asymptomatic severe acute respiratory syndrome-coronavirus 2 (SARS-CoV-2) carrier, or alternatively her second test result was a false-positive. However, the latter explanation is less likely as 5 of her relatives developed COVID-19 after interactions with her.

\section{How Reliable and Comparable Are Test Kits?}

A laboratory test commonly used to diagnose COVID-19 is real-time polymerase chain reaction (RT-PCR), which detects the presence of viral nucleic acids. This is a highly specific and sensitive assay which has been demonstrated to be superior in detecting novel coronavirus (nCoV) than other assays. ${ }^{10}$ Probes are designed to bind to unique sequences in the pathogen for amplification and detection. Currently, diagnostic kits come from different sources, and different kits have different levels of test sensitivity and specificity. If the result of the RT-PCR test is positive for coronavirus, the sample can be sent for genome sequencing to confirm the findings. Patients are deemed to have a positive test if the genetic sequence of the virus in their blood or respiratory tract sample has a "high degree"of similarity with that of the virus. False-positives can only arise if there is contamination of the sample. This could arise during multiple sample collection and cross contamination. False-negatives are a more likely outcome which could be due to the time or site of sampling (e.g. viral load is below the limit of detection of the assay) or degradation of viral ribonucleic acid (RNA) during transport and storage. Xie et $\mathrm{al}^{11}$ evaluated the effectiveness of PCR methods and found that viral nucleic acid could be detected in oropharyngeal swab samples ( $9 / 19$ positive patients) and also stool samples ( $8 / 9$ positives). None of the positive results were identified in blood and urine samples.

One example of a RT-PCR test is the CDC 2019-nCoV RT-PCR Diagnostic Panel, which contains 2019-nCoV N1, 2019-nCoV_N2 and 2019-nCoV_N3 primers and probes that target the nucleocapsid $(\overline{\mathrm{N}})$ gene (designed for universal detection of SARS-like coronavirus as well as specific detection of the 2019-nCoV); RT primers and probes targeting the Human RNase P gene; and nCoVPC, the 2019-nCoV positive control used in the assay. ${ }^{12}$ This test is not yet approved for use by the United States Food and Drug Administration (FDA). However, an Emergency Use Authorization (EUA) for this test is supported by the Secretary of Health and Human Service's declaration that circumstances exist to justify the use of in vitro diagnostics (IVD) under EUA for the detection and/or diagnosis of 2019-nCoV. ${ }^{12}$ An IVD made available under an EUA has 
not undergone the same type of review as an FDA-approved IVD. There has been a report of unreliable kits, where a number of faulty test kits were sent out by United States health authorities to laboratories across the country. ${ }^{13}$ RTPCR assays that target the RNA-dependent RNA polymerase $(\mathrm{RdRp}) /$ helicase (Hel) genes of SARS-CoV-2 have been recently shown to be more sensitive and specific than those that target the spike $(\mathrm{S})$ and nucleocapsid $(\mathrm{N})$ genes. ${ }^{14}$

Determining the levels of specific antibodies to SARS$\mathrm{CoV}-2$ is becoming possible and a rapid point-of-care test has been reported..$^{15}$ This assay uses a recombinant receptor binding domain of SARS-CoV-2 Spike Protein in a lateral flow immunoassay format. Xiang et $\mathrm{al}^{16}$ also compared ELISA IgG and IgM antibodies colloidal goldimmunochromatographic assay with RT-PCR. Best results were obtained by combining IgM and $\mathrm{IgG}$ responses. The limited data to date indicates that immunoassay sensitivity is $83-88 \%$ compared to around $50 \%$ with RT-PCR.

Details of seroconversion of infected patients are largely unknown; however, it has been reported that IgM antibodies were detected 3-6 days after infection with SARS-CoV and $\mathrm{IgG}$ after 8 days. In addition, levels of cross-reaction with closely related $\mathrm{CoV}$ will need to be controlled for. A number of companies are developing immunoassaybased kits (e.g. Snibe Diagnostic received a CE Mark for their Maglumi 2019-nCoV [SARS-CoV-2] IgM/IgG kits recently). ${ }^{17}$ Ultimately, neutralising antibody assays will be valuable but will require level 3 biosafety capacity.

\section{Are Laboratory Facilities Certified and is the Laboratory Manpower Adequately Trained?}

In addition to the availability and quality of test kits, laboratory personnel should be adequately trained to perform these tests accurately. For example, the CDC 2019-nCoV RT-PCR Diagnostic Panel is only authorised for use in qualified laboratories designated by the CDC as qualified, and certified under the Clinical Laboratory Improvement Amendments to perform high complexity tests. ${ }^{18}$

Many laboratories throughout the developing world may not have such capability, and there is a rush to develop such expertise and capacity. ${ }^{19}$ The WHO has activated an international network of 16 referral laboratories that can support national efforts in confirming new cases. ${ }^{20}$

\section{How Are the Results Interpreted?}

RT-PCR is widely used in diagnostic virology and has yielded few false-positive outcomes. ${ }^{21}$ A negative test result indicates that the viral RNA was not identified in the specimen above the limit of detection, but does not exclude the possibility of a false-negative test. False-negative tests should be considered if the patient's recent exposures or clinical presentation indicate that COVID-19 is likely, and diagnostic tests for other causes of illness are negative. In such cases, retesting should be considered. ${ }^{12}$

\section{Conclusion}

Accurate diagnosis of COVID-19 is extremely important for clinical management of cases, for early institution of preventive health measures such as isolation and contact tracing for quarantine measures and for understanding the pattern of disease transmission.

Asignificant proportion of cases are presently undiagnosed in many countries. This is probably due to limited testing of people due to restrictive case definitions of "suspect cases" in the early stages of the outbreak, where laboratory test kits are scarce, and testing is often only limited to "suspect cases". The fewer the laboratory tests are done, the fewer would be the number of confirmed cases and the larger the proportion of undiagnosed cases. As more laboratory test kits become available and laboratory testing is more widely done, the proportion of undiagnosed cases will decrease.

Counting the number of cases (including mild cases) is necessary in order to understand the pattern of disease transmission and for calibration of the epidemic response. However, the variability of case definitions, the populations that are screened (which will vary by location and timeline of the outbreak), testing techniques and interpretation of laboratory results will affect the number of cases enumerated. Given the above, the reliability of epidemiological characterisation of the disease - in terms of disease counts and comparability of numbers within and between countries - can never be completely accurate and unambiguous. Fortunately, it is often true that a close approximation of the true figures would suffice for adequate public health responses.

\section{REFERENCES}

1. World Health Organization. Remarks by Dr Michael Ryan, Executive Director, WHO Health Emergencies Programme at Media Briefing on COVID-19 on 13 February 2020. Available at: https://www.who.int/ news-room/detail/13-02-2020-remarks-by-dr-michael-ryan-executivedirector-who-health-emergencies-programme-at-media-briefing-oncovid-19-on-13-february-2020. Accessed on 22 February 2020.

2. Channel NewsAsia. China Changes Method of Counting COVID-19 Patients Again. 20 February 2020. Available at: https:/www. channelnewsasia.com/news/asia/coronavirus-covid19-china-changedmethod-counting-infected-12455084. Accessed on 22 February 2020.

3. Dorigatti I, Okell L, Cori A, Imai N, Baguelin M, Bhatia S, et al. Report 4: Severity of 2019-Novel Coronavirus ( $\mathrm{nCoV}$ ). WHO Collaborating Centre for Infectious Disease Modelling MRC Centre for Global Infectious Disease Analysis J-IDEA Imperial College London. Available at: https://www.imperial.ac.uk/media/imperial-college/medicine/sph/ide/ gida-fellowships/Imperial-College-COVID19-severity-10-02-2020.pdf. Accessed on 22 February 2020. 
4. Wang D, Hu B, Hu C, Zhu F, Liu X, Zhang J, et al. Clinical characteristics of 138 hospitalized patients with 2019 novel Coronavirus-infected pneumonia in Wuhan, China. JAMA 2020;Feb 7. doi: 10.1001/ jama.2020.1585.

5. Novel Coronavirus Pneumonia Emergency Response Epidemiology Team. The epidemiological characteristics of an outbreak of 2019 novel coronavirus diseases (COVID-19) in China. Zhonghua Liu Xing Bing Xue Za Zhi 2020;41:145-51.

6. Bai Y, Yao L, Wei T, Tian F, Jin DY, Chen L, et al. Presumed asymptomatic carrier transmission of COVID-19. JAMA 2020; Feb 21. doi: 10.1001/ jama.2020.2565

7. Public Health England. COVID-19: Laboratory Investigations and Sample Requirements for Diagnosis. Updated 17 February 2020. Available at: https://www.gov.uk/government/publications/wuhan-novel-coronavirusguidance-for-clinical-diagnostic-laboratories/laboratory-investigationsand-sample-requirements-for-diagnosing-and-monitoring-wn-covinfection. Accessed on 22 February 2020.

8. World Health Organization. Interim Guidance. Global Surveillance for Human Infection With Coronavirus Disease (COVID-2019). 31 January 2020. Available at: https:/www.who.int/publications-detail/globalsurveillance-for-human-infection-with-novel-coronavirus-(2019-ncov). Accessed on 22 February 2020

9. Centers for Disease Control and Prevention. Coronavirus Disease 2019 (COVID-19). Frequently Asked Questions and Answers. Page last reviewed 15 February 2020. Available at: https://www.cdc.gov/ coronavirus/2019-ncov/faq.html. Accessed on 22 February 2020.

10. Lin C, Ye R, Xia YL. A meta-analysis to evaluate the effectiveness of real-time PCR for diagnosing novel coronavirus infections. Genet Mol Res 2015;14:15634-41.

11. Xie C, Jiang L, Huang G, Pu H, Gong B, Lin H, et al. Comparison of different samples for 2019 novel coronavirus detection by nucleic acid amplification tests. Int J Infect Dis 2020;93:264-7.

12. Centers for Disease Control and Prevention. Fact Sheet for Healthcare Providers. CDC - 2019-nCoV Real-Time RT-PCR Diagnostic Panel. 4 February 2020. Available at: https://www.cdc.gov/coronavirus/2019ncov/downloads/Factsheet-for-Healthcare-Providers-2019-nCoV.pdf. Accessed on 22 February 2020.

13. Channel NewsAsia. US Health Authority Shipped Faulty Coronavirus Test Kits Across Country: Official. 13 February 2020, 05:55 am. Updated
13 February 2020, 11:02 am. Available at: https://www.channelnewsasia. $\mathrm{com} /$ news/world/covid19-coronavirus-united-states-faulty-testkits-12429566. Accessed on 22 February 2020.

14. Chan JF, Yip CC, To KK, Tang TH, Wong SC, Leung KH, et al. Improved molecular diagnosis of COVID-19 by the novel, highly sensitive and specific COVID-19-RdRp/Hel real-time reverse transcription-polymerase chain reaction assay validated in vitro and with clinical specimens. J Clin Microbiol2020;Mar4 pii: JCM.00310-20. doi: 10.1128/JCM.00310-20.

15. Li Z, Yi Y, Luo X, Xiong N, Liu Y, Li S, et al. Development and clinical application of a rapid IgM-IgG combined antibody test for SARS-CoV-2 infection diagnosis. J Med Virol 2020;Feb 27. doi: 10.1002/jmv.25727.

16. Xiang J, Yan M, Li H, Liu T, Lin C, Huang S, et al. Evaluation of enzymelinked immunoassay and colloidal gold-immunochromatographic assay kit for detection of novel coronavirus (SARS-Cov-2) causing an outbreak of pneumonia(COVID-19). medRxiv; doi 10.1101/2020.02.27.20028787.

17. 360DX. Snibe Diagnostic Receives CE Mark for SARS-CoV-2 Kits, Immunoassay System. Available at: https:/www.360dx.com/regulatorynews-fda-approvals/snibe-diagnostic-receives-ce-mark-sars-cov-2-kitsimmunoassay-system\#.Xn3qnuozb3g. Accessed on 7 March 2020.

18. Centers for Disease Control and Prevention. CDC Tests for COVID-19. Page last reviewed 15 February 2020. Available at: https://www. cdc.gov/coronavirus/2019-ncov/about/testing.html. Accessed on 22 February 2020.

19. Channel NewsAsia. Countries Rush to Build Diagnostic Capacity As Coronavirus Spreads. 11 February 2020, 05:42 am. Updated 11 February 2020, 05:45 am. Available at: https://www.channelnewsasia.com/news/ world/countries-rush-to-build-diagnostic-capacity-as-coronavirusspreads-12420064. Accessed on 22 February 2020.

20. World Health Organization. Specimen Referral for $2019 \mathrm{nCoV}$ - Operational Details of Referral Laboratories. Last updated 10 February 2020. Available at: https://www.who.int/docs/default-source/ coronaviruse/who-appointed-2019-ncov-referral-laboratories-7february-2020.pdf?sfvrsn=c3fa3ec3_4. Accessed on 22 February 2020.

21. Corman VM, Landt O, Kaiser M, Molenkamp R, Meijer A, Chu DKW, et al. Detection of 2019 novel coronavirus (2019-nCoV) by real-time RT-PCR. Euro Surveill 2020 Jan;25(3). doi: 10.2807/1560-7917. ES.2020.25.3.2000045. 